\title{
The impact of the program of basketball, volleyball and handball on the situation-motorized capability of the first classes of the elementary school
}

\author{
Nikšić E. ${ }^{1 \mathrm{ABCDE}}$, Beganović E. ${ }^{2 \mathrm{ABCDE}}$, Joksimović M. ${ }^{3 \mathrm{ADE}}$ \\ ${ }^{1}$ Faculty of Educational Sciences, University of Sarajevo, Bosnia and Herzegovina \\ ${ }^{2}$ Faculty of Sport and Physical Education, University of Sarajevo, Bosnia and Herzegovina \\ ${ }^{3}$ Football club National, Podgorica, Montenegro
}

Authors' Contribution: A - Study design; B - Data collection; C - Statistical analysis; D - Manuscript Preparation; E - Funds Collection.

\begin{abstract}
Purpose:

Determining the state of the student and his situational-motor skills, and their comparison, enables the teacher to control his work in an immediate manner, and he programmes and implements well planned and additional contents in order to realize the set goals. In order to achieve these functions, it is necessary to determine the current state of the students, as well as the state after the implemented specific contents and programs. The aim of the research was to determine the partial changes (differences) of situationalmotor abilities that were created under the influence of a four-month program of basketball, volleyball and handball in the pupils of the fifth grades of elementary school.

Material: $\quad$ The study included $\mathrm{N}=106$ class $\mathrm{V}$ students, aged 10 to 11 years. The sample of respondents was divided into two subgroups, an experimental group (53 students), who conducted physical and health education classes according to the modified plan and program of sports games (basketball, volleyball and handball) for a half-year and control group (53 students) who attended regular classes from physical and health culture according to the current curriculum. 9 variables were used to assess situational-motor abilities. Descriptive statistics and discriminatory analysis were used to determine the changes (differences) in situational-motor skills.

Results: $\quad$ The results of the research at the descriptive level showed certain differences between the groups in the final versus the initial measurements on the individual variables of situational motors. Based on the results of the discriminatory analysis, the relative contribution of each of the variables of the situational motor in the final measurement is seen. The SMKVLS (Running the ball with your hand in the slalom) and SMRBLZ (Throwing the ball against the wall for 30 seconds) variables are the biggest contributors, and the smallest SMOGCPM (Aiming the goal over the net from the basic stand) and SMOSD (Lower frontal serve).

Conclusions: $\quad$ Physical and health culture in schools has the primary task of influencing positive transformation processes in all dimensions of students by applying appropriate content.

Keywords: experimental program, sports games, situational-motor skills, pupils.
\end{abstract}

\section{Introduction}

Previous research on the student population shows that properly programmed teaching or training processes contribute and help to the overall correct growth and development of children $[1,2,3,4]$. For this reason, it is very important to monitor the effects of teaching or training processes in each phase by appropriate instruments. All of this presupposes relevant information on the basis of which it would be more precisely determined, first of all, the initial state, and in the further process and adequate monitoring of the effects of the programmed activities applied, and afterwards, the final state.

Determining the condition of the students, but also of the group as a whole, allows the teacher to control their work, as well as the planned contents that are implemented to achieve the set goals. In order to achieve the expected results, it is necessary to determine the initial state of the students, at the beginning of the year for programming work, and finally for analyzing the performance of work. Thus, the determined initial state and the predicted

(c) Nikšić E., Beganović E., Joksimović M., 2020

doi:10.15561/26649837.2020.0206 desired final state of the students would enable the teachers to determine the state of the students during the year, from the initial to the final state, and in the end to the final analysis and assessment of the effectiveness of the applied model of training, i.e. basketball, volleyball and handball content [5].

Nikšić et al. [5] in his master's thesis on the sample of 50 boys $12-14$ years old who are involved in the training process and 50 examinees of the same age who are not involved in the football training process determines the level of differences in basic and situational motor skills. The greatest quantitative differences in space with situational motor skills have been achieved by the following variables: the speed of running the ball at $20 \mathrm{~m}$ with start from the place, the speed of running the slalom, running speed with right angle direction and the ball speed per semicircle for the benefit of children who deal with systematic sports, in this case football.

Ražanica [6], on a sample of 127 high school students, aged 15 to 17 years, determines the relations and magnitudes of the influence of some motor skills on the success of performing situational motor tests in sports 
games. In the area of motor skills, eight tests were carried out according to Eurofit, which evaluated five latent hypothetically defined motor dimensions. The results of the regression analysis suggest that the success in sports games is guaranteed by those students whose ability lies in the effectiveness of the regulation system for movement.

Lakota [7] in his master's thesis on the sample of 82 handball players 11-14 years of age tried to determine qualitative and quantitative changes in basic situational motor skills created under the influence of the threemonth program of handball. A 52-course handball program produced statistically significant changes in the treated areas.

Hadžikadunić [8], on a sample of 146 male students, identified transformation processes under the influence of programmed physical and health education for 69 hours of instruction in basic motor, situational and motor skills and functional abilities of students of the eighth grade. A system of variables of 8 tests for basic motor abilities and 9 tests for the assessment of specific motor abilities was used, and one test for the evaluation of functional abilities. It was found that programmed teaching has a positive effect on improving basic motor, situational and functional abilities between two measurements.

Bajramović et al. [9] on a sample of 103 footballers aged 12-14 years tried to determine the levels of transformation of motor skills and the success of footballers under the influence of six-month programmed work. Using the t-test for dependent samples at the univariate level, it was noted that the football program caused a number of significant changes after the final measurement. Using factor or discriminatory analysis at the multivariate level, the author concludes that significant global quantitative changes have occurred in the space of situational motor skills and success in the game, while weak changes were observed in the area of basic motor skills.

Lakota et al. [10] determined the qualitative level of transformation of situational motor skills in handball players aged 11-14 years in duration of three months. The sample consisted of 82 male entities. Factor analysis isolated $73 \%$ of the total variability. The results indicate that qualitative changes have been shown when hitting the goal with the ball, as well as the speed of movements related to ball control.

Mladenović [11], in his paper "Structural changes in sports games in the teaching of physical education" on the sample of 152 students, implemented the contents of sports games in the teaching of physical education for the duration of one school year. By applying the treatment, the level of structural changes in basic and specific motors was attempted. The results of this program have shown a general, systematic, continuous reconstruction of general and specific motor abilities, therefore the transfomation process is responsible, although not in all situations to the same extent. The worst effects are recorded in the case of football.

Malacko, \& Pejić [12] have studied the changes of biomotor pupils aged 11 years under the influence of experimental program of sports games in relation to the standard bodybuilding program. The sample was made up of 252 male students, who were divided into a control and experimental group. A system of 33 variables (12 morphological and 21 for estimation of motor and functional abilities) was used. The experimental program was saturated with the contents of sports games. The results indicate that the morphological system contributes to the same differentiation of the group, while the experimental group showed better results in the motor space, of which 14 variables showed a statistically significant difference in 13 variables, the experimental group was better.

Džumhur [13], investigated changes in motor skills and performance in a small soccer game using the situational method of work. On a sample of 81 subjects, aged 12-14 years. He established that the program with the application of the situational method of work revealed changes in the coordination segment, the speed of movement and the segment of equilibrium. It has positively influenced the improvement of situational performance in the soccer game.

The aim of the research was to determine the partial changes (differences) of situational-motor abilities that were created under the influence of a four-month program of basketball, volleyball and handball in the pupils of the five grades of elementary school.

\section{Material and Methods \\ Participants}

The study was conducted on a sample of $n=106$ pupils in the fifth grade, female, aged 10 to 11 years, clinically and mentally healthy, and with no pronounced morphological and locomotor impairments. The sample of respondents was divided into two subgroups, an experimental (53 pupils) and a control group (53 students). The experimental group conducted classes according to the changed curriculum. The program included sports games from handball, basketball and volleyball. The control group carried out the teaching according to the current curriculum.

\section{Research Design}

The variables used in this study consisted of 9 situational-motor variables: basketball 3 variables, volleyball 3 variables and handball 3 variables.

- BASKETBALL (Throwing the ball with both hands against the wall and catching it for 30 seconds SMKBLRZ; Running the ball with your hand in the slalom - SMKVLS; Throwing the ball into the basket for 30 seconds - SMKBLK).

- VOLLEYBALL (Lower frontal serve - SMOSD; Aiming the goal over the net from the basic stand SMOGCPM; Forearm pass in circle for 30 seconds - SMOOLPK).

- HANDBALL (Throwing the ball against the wall for 30 seconds - SMRBLZ; Running the ball in the slalom - SMRVLS; Performing sevens - SMRIS).

Work program

During the first semester, three teaching units were processed as part of regular classes: athletics, basketball and volleyball. A total of 35 teaching hours of regular 
physical and health education were held, of which 12 hours of athletics, 12 hours of basketball, 11 hours of volleyball. The program of additional classes through the basketball, volleyball and handball sports games consisted of a modified curriculum from basketball: adding and catching balls from basketball, running a ball with a stop, a basketball technique, a low-lead technique, a kick-off practice with zipper positions, zigzag guiding, one - handed addition, moving the ball with arms in motion, running the ball with stop in the position of the shot, ball manipulation, pivoting technique with the ball, straight line guidance from high to low, and vice versa. From the volleyball sports, some teaching units worked, for example: hammer hit, passing with fingers over the net, training a school service, mini volleyball, hammering overhead, refusing to throw a ball out of the wall, adding alternate fingers - a hammer, school service with six and nine meters, jumping with both legs from dockyards on the net, shooting a basket with a hammer. From the handball, the teaching units worked as follows: foreclosure, lateral addition, jumping, kicking on the goal, slalom, handball, straight tracking, mini handball, goal kicking - seven, manipulation with a handball, Shade Adding, Adding To The Triples Game 1: 1 Shot on goal. Only girls were involved in this program and for this reason football was not taken as a spot game.

Statistical Analysis

A descriptive statistical procedure was applied in the data processing process.
The following descriptive parameters are calculated:

- Arithmetic mean (Mean);

- Standard deviation (Std. Deviation).

At the multivariate level, changes were made:

- A discriminatory analysis was used to determine the changes and differences between the experimental and control group under the influence of the experimental program in the situational-motor tests.

\section{Results}

Display of the collected data by the given characteristics.

Table 1 above presents the average values and measurements of the deviation of the results of the control and experimental group on the variables of the situational motors in the initial measurement. At the descriptive level, there are noticeable differences between groups.

Table 2 above gives an overview of the average values of the control and experimental group on the individual variables of the situational motor in the final measurement. In this case, the differences between the groups are noticeable at the descriptive level.

In the following section, a discriminatory analysis was made to determine the intergroup differences in the individual components of situational motors in the initial and final measurement.

Based on the results of the discriminatory analysis, one significant discriminatory function was identified, the coefficient of the canonical coalition being 0.60 . It can be said that this is a significant and moderate correlation

Table 1. Values of arithmetic meanings and standard deviations of situational-motors initial measurement

\begin{tabular}{llllll}
\hline Variables & Group & $\mathbf{N}$ & Mean & Std. Deviation & Std. Error Mean \\
\hline \multirow{2}{*}{ SMKBLRZ } & 1 & 53.00 & 23.38 & 5.20 & 0.71 \\
& 3 & 53.00 & 22.19 & 5.89 & 0.81 \\
SMKVLS & 1 & 53.00 & 10.32 & 1.39 & 0.19 \\
& 3 & 53.00 & 11.41 & 2.64 & 0.36 \\
SMKBLK & 1 & 53.00 & 4.17 & 2.52 & 0.35 \\
& 3 & 53.00 & 3.83 & 2.42 & 0.33 \\
SMOSD & 1 & 53.00 & 6.72 & 3.07 & 0.42 \\
& 3 & 53.00 & 6.83 & 3.34 & 0.46 \\
SMOGCPM & 1 & 53.00 & 4.34 & 2.36 & 0.32 \\
& 3 & 53.00 & 4.98 & 2.23 & 0.31 \\
SMOOLPK & 1 & 53.00 & 18.34 & 4.93 & 0.68 \\
& 3 & 53.00 & 20.13 & 6.65 & 0.91 \\
SMRBLZ & 1 & 53.00 & 21.42 & 3.86 & 0.53 \\
& 3 & 53.00 & 23.32 & 4.55 & 0.63 \\
SMRVLS & 1 & 53.00 & 11.05 & 1.73 & 0.24 \\
SMRIS & 3 & 53.00 & 11.90 & 2.33 & 0.32 \\
& 1 & 53.00 & 3.36 & 1.67 & 0.23 \\
\hline
\end{tabular}

NOTES: 1 - Control group, 3 - Experimental group; SMKBLRZ - Throwing the ball with both hands against the wall and catching it for 30 seconds; SMKVLS - Running the ball with your hand in the slalom; SMKBLK - Throwing the ball into the basket for 30 seconds; SMOSD - Lower frontal serve; SMOGCPM - Aiming the goal over the net from the basic stand; SMOOLPK - Forearm pass in circle for 30 seconds; SMRBLZ - Throwing the ball against the wall for 30 seconds; SMRVLS - Running the ball in the slalom; SMRIS - Performing sevens. 
Table 2. Values of arithmetic meanings and standard deviations of situational-motors final measurements

\begin{tabular}{llllll}
\hline Variables & Group & N & Mean & Std. Deviation & Std. Error Mean \\
\hline \multirow{2}{*}{ SMKBLRZ } & 2 & 53.00 & 26.66 & 4.02 & 0.55 \\
SMKVLS & 4 & 53.00 & 24.89 & 5.59 & 0.77 \\
& 2 & 53.00 & 9.24 & 1.00 & 0.14 \\
SMKBLK & 4 & 53.00 & 10.21 & 1.97 & 0.27 \\
& 2 & 53.00 & 6.58 & 2.56 & 0.35 \\
SMOSD & 4 & 53.00 & 5.51 & 2.85 & 0.39 \\
& 2 & 53.00 & 8.89 & 2.49 & 0.34 \\
SMOGCPM & 4 & 53.00 & 9.06 & 2.94 & 0.40 \\
SMOOLPK & 2 & 53.00 & 6.30 & 2.05 & 0.28 \\
SMRBLZ & 4 & 53.00 & 6.68 & 2.06 & 0.28 \\
SMRVLS & 2 & 53.00 & 21.83 & 5.36 & 0.74 \\
SMRIS & 4 & 53.00 & 24.83 & 7.22 & 0.99 \\
& 2 & 53.00 & 23.45 & 3.94 & 0.54 \\
\hline
\end{tabular}

NOTES: 2 - Control group, 4 - Experimental group

of the structural latent component variants of situational motions in the initial measurement.

According to the value and significance level of the Box test, it can be concluded that there are significant uneven elements matrices of variance-covariance of manifest variables of situational motors in the control and experimental group.

In the described analysis, one discriminatory function was isolated with a canonical correlation value of 0.60 and the value of the characteristic root of 0.57 . On average, the factors of the latent canonical variation, ie, functions can explain $57 \%$ of the variability in the individual situation motors variables.

According to the Willks lambda indicator and the level of significance of the Hi-squared (which is less than $1 \%$ ), it can be concluded that the isolated-discriminating function is statistically significant and that selected manifestation variables of situational motors in the initial measurement significantly contribute to the discriminatory function of the classification prediction of the control group and the experimental group in the initial measurement.

Table 3. Box's M test initial measurement

\begin{tabular}{lll}
\hline Box's $M$ & & 90.56 \\
\hline $\mathrm{F}$ & Approx. & 1.83 \\
& $\mathrm{df1}$ & 45.00 \\
& $\mathrm{df2}$ & 35532.53 \\
& Sig. & 0.00 \\
\hline
\end{tabular}

Table 4. Significance of isolated discriminant functions initial measurement

\begin{tabular}{llllll}
\hline Function & Eigenvalue & \% of Variance & Cumulative \% & $\begin{array}{l}\text { Canonical } \\
\text { Correlation }\end{array}$ \\
\hline dimension0 & 1 & $.57^{\mathrm{a}}$ & 100.00 & 100.00 & .60 \\
\hline
\end{tabular}

Table 5. Wilks' Lambda initial measurement

\begin{tabular}{|c|c|c|c|c|}
\hline Test of Function(s) & Wilks' Lambda & Chi-square & df & Sig. \\
\hline dimension0 & 0.64 & 44.96 & 9.00 & 0.00 \\
\hline
\end{tabular}


Table 6. The structure of the discriminatory function of the initial measurement

\begin{tabular}{ll}
\hline Variables & Function \\
& $\mathbf{1}$ \\
\hline SMKVLS & 0.35 \\
SMRBLZ & 0.30 \\
SMRVLS & 0.28 \\
SMOOLPK & 0.20 \\
SMRIS & 0.20 \\
SMOGCPM & 0.19 \\
SMKBLRZ & -0.14 \\
SMKBLK & -0.09 \\
SMOSD & 0.02 \\
\hline
\end{tabular}

Table 7. Centroids group initial measurement

\begin{tabular}{lll}
\hline GROUP & Function \\
& & 1 \\
\hline \multirow{2}{*}{ di } & 1 & -0.75 \\
& 3 & 0.75 \\
\hline
\end{tabular}

Table 6 above, it can be seen that some situationalmotors variables in initial measurement in different ways contribute to an isolated discriminative function, and according to individual values of the coefficients. In doing so, the greatest contribution to the maximum intergroup distinction is given by the variables SMKVLS - Handling the ball by hand in the slalom and SMRBLZ - Throwing the ball on the wall for 30 seconds, while the smallest relative contribution has the variables SMKBLK Throwing the ball into the basket for 30 seconds and SMOSD - Service bottom.

When it comes to centroid groups, they represent standardized near-zero variants in the structure of which are differentiated weighted combinations of situational motions variables. Given the centroid value, it can be seen that the cross-sectional criterion is important in the middle between the centroid values and that the centroids are fairly uniform. This means that there is no difference in the correct classifications and distinctions between the control and the experimental group, as in both groups the percentages of the correct classification are uniform.

In this discriminative analysis, one discriminatory function is also isolated, as can be seen from the following tabular displays.

Table 8. Box's M test final measurement

\begin{tabular}{lll}
\hline Box's $\mathbf{M}$ & & $\mathbf{6 6 . 5 1}$ \\
\hline $\mathrm{F}$ & Approx. & 1.34 \\
& df1 & 45.00 \\
& df2 & 35532.53 \\
& Sig. & 0.06 \\
\hline
\end{tabular}

The Box's test is not statistically significant, and it can be said that in the final measurement, the elements of the variance-covariance matrix of the components of the situational motor are mutually uniform, while in the previous, initial measurements they are not. This indicates a higher homogeneity of the groups in the situation after the work program in relation to the measurement before the work program.

An isolated discriminating function has a characteristic root value greater than 1 which indicates that the factors of the latent structure of a discriminant function can explain, on average, the overall variability of one manifest variable of situational motoring. A canonical correlation of 0.71 indicates a high connection between the variants of latent factors. This correlation is higher than in the initial measurement, which indicates a greater possibility for the model to achieve better distinctions between groups.

A model with one discriminative function is statistically significant, which is confirmed by the statistically significant Willks Lmbda expression and Hisquare.

\section{Discussion}

Previous research on the student population shows that properly programmed teaching or training processes contribute and help to the overall correct growth and development of children [14 2,4]. Therefore, it is very important to monitor the effects of teaching or training processes with appropriate instruments.

Hadžikadunić [8], on a sample of 146 male students, identified transformation processes under the influence of programmed physical and health education for 69 hours of instruction in basic motor, situational and motor skills and functional abilities of students of the eighth grade. A system of variables of 8 tests for basic motor abilities and 9 tests for the assessment of specific motor abilities was used, and one test for the evaluation of functional abilities. It was found that programmed teaching has a positive impact on improving basic motor, situational and functional abilities between two measurements (initially and final).

Hadžikadunić [8] determined the qualitative level of transformation of situational motor skills in handball players aged 11-14 years in duration of three months. The sample consisted of 82 male subjects. Factor analysis isolated $73 \%$ of the common variability.

The results indicate that qualitative changes have been shown when hitting the goal with the ball, as well as the speed of movements related to ball control.

Hadžikadunić [8], in his paper "Structural changes in sports games in the teaching of physical education" on the sample of 152 students, implemented the contents of sports games in the teaching of physical education for the duration of one school year. By applying the treatment, the level of structural changes in basic and specific motors was attempted. The results of this program have shown a general, systematic, continuous reconstruction of general and specific motor abilities, therefore the transformation process is responsible, although not in all situations to the same extent. The worst effects are recorded in the case of football. 
Table 9. Significance of isolated discriminatory functions Final Measurement

\begin{tabular}{llllll}
\hline Function & \multicolumn{2}{l}{ Eigenvalue } & \% of Variance & Cumulative \% & Canonical Correlation \\
\hline dimension0 & 1 & $1.01^{\mathrm{a}}$ & 100.00 & 100.00 & .71 \\
\hline
\end{tabular}

Table 10. Wilks' Lambda final measurement

\begin{tabular}{llllll}
\hline Test of Function(s) & Wilks' Lambda & Chi-square & df & Sig. \\
\hline dimension0 & 1 & 0.50 & 69.59 & 9.00 & 0.00 \\
\hline
\end{tabular}

Table 11. The structure of the discriminating function of the final measurement

\begin{tabular}{ll}
\hline Variables & $\begin{array}{l}\text { Function } \\
\mathbf{1}\end{array}$ \\
\hline SMKVLS & 0.31 \\
SMRBLZ & 0.29 \\
SMOOLPK & 0.24 \\
SMRIS & 0.23 \\
SMKBLK & -0.20 \\
SMKBLRZ & -0.18 \\
SMRVLS & 0.13 \\
SMOGCPM & 0.09 \\
SMOSD & 0.03 \\
\hline
\end{tabular}

Malacko, \& Pejić [12], investigated the changes of pupils aged 11 years under the influence of the experimental program of sports games in relation to the standard program of physical education. The sample was made up of 252 male students, who were divided into a control and experimental group. A system of 33 variables (12 morphological and 21 for estimation of motor and functional abilities) was used.

The experimental program was saturated with the contents of sports games. The results indicate that the morphological system contributes to the equal differentiation of the group, while the experimental group showed better results in the motor space, of 14 variables, which showed a statistically significant difference in 13 variables, was an experimental group.

Based on the results of the arithmetic meanings in the tests for the assessment of situational-motor abilities, at the beginning and at the end of the programmed exercise from volleyball, and on the basis of the significance of the changes tested with the T-test for the dependent samples, it is clearly visible that the programmed exercise from volleyball produced significant partial effects. In tests for assessment of situational-motor abilities, presented in this research by the variables SMJAPT - Japanese test, SMTESJ - seating test, SMPRSE - precision of serving, SOPKNZ - rejection of the ball in a circle on the wall and SOPPOZ - rejection of the ball with forearms on the wall, there is a statistically significant positive shift in the value of the arithmetic mean in all tested variables at the final measurement at the statistically most significant level [14].
Tabela 12. Centroids group final measurement

\begin{tabular}{lll}
\hline GROUP & & Function \\
& & 1 \\
\hline di & 1 & -0.99 \\
& 3 & 0.99 \\
& & \\
\hline
\end{tabular}

Analyzing the results of the final with respect to the initial situation of situational - motor abilities, there has been a rewriting of the variables and an increase in the number of factors, which shows that there have been qualitative changes in the structure, as well as the transformation of some numerical indicators of situational - motor abilities of the respondents. Looking at the whole, the program of regular and additional teaching with its contents and training exercises and loads had a significant influence on the qualitative changes in situational and motor skills [15].

\section{Conclusion}

This research was conducted with the aim of determining the partial change (difference) of situationalmotor skills created by the influence of a four-month program of basketball, volleyball and handball in the pupils of the five grades of elementary school. The sample of respondents included 106 pupils in the fifth grade, aged 10 to 11 years, clinically and mentally healthy, and with no pronounced morphological and locomotor impairments. The sample of respondents was divided into two subgroups, an experimental group (53 pupils), who carried out physical education classes according to the modified plan and program of sports games (basketball, volleyball and handball) for a semester and a control group (53 pupils) who attended regular classes from physical education according to the current curriculum. The variables applied in this study consisted of 9 variables for assessing situational motor performance in sports games and variables for assessing situational motor performance 
from basketball (Throwing a hand with a hand on the wall and capturing for 30 seconds - SMKBLRZ; Handling the ball by hand in the slalom - SMKVLS; Throwing the ball into the basket for 30 seconds - SMKBLK), variables for assessing situational motor performance from volleyball (Service bottom chess - SMOSD; Targeting the target through the net from the base bet - SMOGCPM; SMOOLPK), variables for assessing situational motor performance from the handball (Throwing the ball on the wall for 30 seconds - SMRBLZ; Running the slalom ball - SMRVLS; Performing the seven-meter - SMRIS). Descriptive statistics and discriminatory analysis were used to determine the changes in the situational-motor abilities. The results of the research at the descriptive level showed certain differences between the groups in the final versus the initial measurements on the individual variables of situational motors. Based on the results of the discriminatory analysis, the relative contribution of each of the variables of the situational motor in the

\section{References}

1. Skender N. Transformation processes of motor skills and anthropological characteristics under the influence of a seven-month treatment in third and fourth grade students. [Doctor's thesis]. Sarajevo: Faculty of Sport and Physical Education; 2003.

2. Tabaković M, Skender N, Turković S. Quantitative differences in motor skills of pupils of lower grades of primary school after realization of certain programs, Homosporticus, 2006; 9(2): 44-50.

3. Hodžić M, Mekić M. Influence of the transformation program of additional hours of physical education on physical motor development of children aged 14-16, Proceedings from the IV International Conference "Sport Management", 2008. P. 421-441.

4. Ćeleš N. The influence of programmed exercise on the transformation of morphological characteristics, motor skills and the adoption of elements of volleyball technique. [Doctoral thesis]. Tuzla: Faculty of Physical Education and Sport; 2009.

5. Nikšić E, Rašidagić F, Mahmutović I, Mujčić A. Ball games in the function of the development of a child.Sarajevo: Faculty of educational sciences University of Sarajevo; 2015. P. 24-50.

6. Ražanica F. Connection of some motor skills and conative regulatory mechanisms with success in sports games among high school students. [Master's thesis]. Sarajevo: FASTO; 2004.

7. Lakota R. Effects of programmed work on transformation of basic-motor and situational-motor skills of handball players. [Master's thesis]. Sarajevo: Faculty of Sport and Physical Education; 2006. final measurement is seen. The SMKVLS and SMRBLZ variables have the greatest contribution, and the smallest SMOGCPM and SMOSD. These coefficients indicate the relative contribution of these variables of the model's ability to distinguish between members of one or the other group. Centroid values are higher than in initial measurements, which means that the model has better distinctions than in the initial measurement. Because of this, it can be said that there are indications that the effect of the work program has led to changes in the latent structures of the groups, which lie at the basis of the values of the manifest variables of situational motions. The results obtained can be of benefit to teachers, as well as professors of physical and health culture in conceiving similar programs and their implementation in everyday teaching practice.

\section{Conflicts of Interest}

The authors declare no conflict of interest.
8. Hadžikadunić A. Transformation processes of basic motor, situational motor and functional abilities of pupils of the 8 th grade under the influence of programmed teaching of physical and health education. [Master's thesis]. Sarajevo: Faculty of Sport and Physical Education; 2007.

9. Bajramović I, Talović M, Alić H, Jelesković E. Levels of quantitative changes in the specific-motor skills of footballers under the influence of situational training, Sport Mont. 2008; 6(15, 16, 17):104-109.

10.Lakota R, Talović M, Jeleković E, Bonacin D. Effects of programmed training on transformation of the qualitative level of situational-motor skills of handball players aged 1114 years. Sport Science.2008; 1(2): 60-64.

11. Mladenović M. Structural changes in sports games in the teaching of physical education. Sport Science. 2008; 1(2): 39-43.

12.Malacko J, Pejić A. Changes in the biomotor dimensions of pupils aged 11 years, the experimental program of sports games in relation to the standard program of physical education. Sport Science. 2009; 2(1): 52-61.

13.Džumhur A. The effects of the situational method of work on improving basic motor skills and performance in a small football game in children aged 12-14 years. [Master's thesis]. Sarajevo: Faculty of Sport and Physical Education; 2009.

14.Šmigalović M, Bajrić O, Lolić D. Influence of the volleyball program on the basic and situational motor skills of students aged 13-14 years. Sports Science and Health. 2012; 2(1):3540.

15.Talović M, Mahmutović I, Jelešković E, Alić H. Ormanović $\breve{S}$. Lakota R, et al. Effects of regular and additional teaching of Physical and Health Education on qualitative changes of specific motor skills of pupils. Sportski Logos. 2015; 24-25: $37-44$. 


\section{Information about the authors:}

Nikšić E.; (Corresponding author); http://orcid.org/0000-0002-1849-9693; elvira.beganovic1982@gmail.com; Faculty of Education Science, University of Sarajevo; 71000 Sarajevo, Bosnia and Herzegovina.

Beganović E.; http://orcid.org/0000-0002-5190-6035; edinn.beganovic@hotmail.com; Faculty of Physical Education and Sport, University of Sarajevo, 71000 Sarajevo; Bosnia and Herzegovina.

Joksimović M.; http://orcid.org/0000-0003-4232-5033; nicifor007@outlook.com; Football club National, Ljajkovići bb, 80000 Podgorica, Montenegro.

Cite this article as:

Nikšić $E$, Beganović $E$, Joksimović $M$. The impact of the program of basketball, volleyball and handball on the situation-motorized capability of the first classes of the elementary school. Pedagogy of physical culture and sports, 2020;24(2):85-92.

https://doi.org/10.15561/26649837.2020.0206

This is an Open Access article distributed under the terms of the Creative Commons Attribution License, which permits unrestricted use, distribution, and reproduction in any medium, provided the original work is properly cited (http://creativecommons.org/licenses/by/4.0/deed.en).

Received: 15.10 .2019

Accepted: 15.11.2019; Published: 30.12.2019 